\title{
Motivação para prática do pole dance como atividade física por mulheres
}

\author{
Motivation for pole dance practice as physical activity by women
}

ELIS REgINA LOPES ${ }^{1}$

Thiago Emanuel Medeiros ${ }^{\text {II }}$

Veronica Volski Mattes

'Universidade Estadual do Centro-

-Oeste (UNICENTRO), Guarapua-

va/PR - Brasil.

"Universidade do Estado de Santa

Catarina (UDESC). Florianópolis/

SC - Brasil.
Resumo O presente estudo teve como objetivo avaliar fatores motivacionais para a prática de pole dance em mulheres. Fizeram parte da pesquisa trinta mulheres, com idade média de 31 anos. Como instrumentos foram utilizados um questionário socioeconômico e a validação em português do Exercise Motivations Inventory (EMI-2). Para a análise dos dados, usou-se estatística descritiva (média e desvio padrão). Os resultados evidenciaram que houve maior importância para as dimensões motivacionais "condicionamento físico", "diversão e bem-estar" e "controle de estresse". Em menor destaque apareceu a dimensão "reconhecimento social". Embora haja poucos estudos que discutem a prática de pole dance como exercício físico, conclui-se que, para a amostra pesquisada, a motivação para a prática de pole dance tem sido importante para a manutenção da saúde e condicionamento físico.

Palavras-chave: Motivação. Mulheres. Exercício. Psicologia DO ESPORTE.

ABstraCt The present study aimed to evaluate motivational factors for pole dance practice in women. Thirty women, with a mean age of 31 years, were included in the study. As instruments, a socioeconomic questionnaire and the Portuguese validation of the Exercise Motivations Inventory (EMI-2) were used. Data were analyzed using descriptive statistics (mean and standard deviation). The results showed that there was greater importance for the motivational dimensions "physical conditioning", "fun and well-being" and "stress control". To a lesser extent, the "social recognition" dimension appeared. Although there are few studies that discuss the practice of pole dance as physical exercise, it is concluded that, for the sample studied, the motivation for the practice of pole dance has been important for the maintenance of health and physical conditioning.

Keywords: Motivation. Women. Exercise. Psychology sports.

\section{INTRODUÇÃO}

O exercício físico, praticado de forma sistematizada, faz que o organismo humano melhore a aptidão de forma geral, ocorrendo várias adaptações orgânicas. Desse modo, todo e qualquer exercício físico deve ser incentivado para a população em geral, visando promover melhoria no qua- 
dro de saúde e qualidade de vida. ${ }^{1} \mathrm{O}$ grande desafio dos profissionais da Educação Física e do próprio aluno é conseguir manter uma regularidade na prática do exercício, pois nem todos tomam gosto pela prática escolhida, o que faz as pessoas estarem em uma constante busca por algo novo e que desperte prazer pela prática.

A crescente difusão de informações e imagens a respeito de saúde do corpo e de todas as formas de movimento resulta num aumento pela procura de atividade física, mas não garante a real adesão continuada à mesma. Ou seja, as pessoas iniciam a prática estimulada por vários motivos, mas não conseguem incorporá-la efetivamente no seu cotidiano, deixando-a de lado seguidas vezes. ${ }^{2}$

$\mathrm{Na}$ escolha e na prática regular de exercícios físicos, um dos fatores que influenciam diretamente é a motivação. Segundo Sage, ${ }^{3}$ motivação é o direcionamento do esforço quando o indivíduo procura se aproximar ou é atraído por certas situações. A intensidade do esforço é o quanto uma pessoa coloca em determinada situação que tem ou não tem interesse em executar.

A motivação á caracterizada como um processo ativo, intencional e dirigido a uma meta, que depende da interação de fatores pessoais (intrínsecos) e ambientais (extrínsecos). Uma pessoa é motivada intrinsecamente quando realiza atividades físicas ou pratica esportes por prazer, saúde, bem-estar e satisfação pessoal. Uma pessoa é motivada extrinsecamente quando pratica um esporte ou atividade física por status social, reconhecimento social, aparência física etc. ${ }^{4}$

Há várias teorias que explicam a motivação. Uma delas é a Teoria da Autodeterminação (TAD), que foi proposta por Deci e Ryan
$(1985 ; 2000)$ e expressa a integração de diferentes fatores motivacionais, como intrínsecos, extrínsecos e amotivacionais, que podem influenciar, positivamente, na motivação, quando inseridos em contextos específicos. ${ }^{5}$

A motivação para a prática esportiva depende da interação entre fatores da personalidade, como expectativas, motivos, necessidades e interesses, e fatores do meio ambiente, como facilidades, tarefas atraentes, desafios e influências sociais. ${ }^{6} \mathrm{O}$ nível de motivação, a atratividade e a estimulação estão relacionados a exercícios que a pessoa tenha capacidade suficiente para executar com o mínimo de dificuldade. Além disso, um ambiente adequado e elogios também contam nesse aspecto. ${ }^{7}$

Os motivos que fazem as pessoas procurarem por programas de exercícios físicos mudam com o tempo. Da mesma forma, a razão pela qual as pessoas aderem a programas de exercícios físicos não é a mesma que as faz permanecerem. Nesse sentido, o profissional de Educação Física tem papel fundamental na motivação do aluno, influenciando na sua aderência e permanência no programa. ${ }^{3}$

Da mesma forma, é crescente o número de novas práticas no mercado fitness como propostas de atividades físicas inovadoras para a população. O pole dance é um exemplo entre as práticas contemporâneas.

O pole dance é uma prática antiga, mas que está em expansão no Brasil. Pode ser basicamente, definido como "dança no mastro", em que seus praticantes realizam performances que mesclam força, flexibilidade e expressões corporais em uma barra vertical. A atividade ficou popularmente conhecida por ser apresentada em boates ou casas noturnas com expressões sensuais ou erotizadas. ${ }^{8}$ 
Contudo, a origem do pole dance está diretamente ligada à ioga, mais especificamente à prática do mallakhamb. O mallakhamb é uma variação da ioga, muito praticado na Índia, que faz uso de um mastro de madeira vertical com cordas e exige de seus praticantes equilíbrio, força e flexibilidade. ${ }^{9}$

O pole dance ganhou destaque a partir dos anos 1920, na Inglaterra, quando começou a ser praticado em circos. Nessa época, mulheres se apresentavam em performances utilizando postes, que seguravam as tendas circenses, nos intervalos dos números principais. Já o pole dance moderno, como é conhecido hoje, teve início na década de 1990, no Canadá e Estados Unidos da América. Foi adotado como atração circense com grande influência do mastro chinês e usado no Cirque $d u$ Solei, companhia circense com base em Montreal e Quebec, no Canadá, reconhecida mundialmente por seu entretenimento artístico de alta qualidade. ${ }^{10}$ No início do presente século, o pole dance se desenvolveu em gêneros diversos, do exótico ao pole fitness, o último evoluído em 2006, que permitiu às mulheres utilizarem da técnica dentro das suas rotinas diárias de fitness.

Apesar do desenvolvimento do pole dance no Brasil e de sua crescente procura como exercício físico, a produção científica sobre o assunto ainda é escassa. Estudos são necessários para esclarecer os reais benefícios dessa atividade física, bem como, os fatores que levam os praticantes a aderir e permanecer em programas dessa modalidade. Sendo assim, o presente estudo tem por objetivo identificar os motivos que levam à procura e adesão de mulheres às aulas regulares de pole dance como exercício físico.

\section{Metodologia}

O estudo foi desenvolvido de forma quantitativa e descritiva. Pesquisas, dessa natureza, traduzem em números as opiniões e informações, a fim de classificá-las e analisá-las por meio de recursos e técnicas estatísticas. $^{11}$

A pesquisa foi realizada em um studio/ academia de dança de uma cidade do interior do Estado do Paraná. Segundo dados fornecidos pela proprietária, o local possui o pole dance como modalidade há cinco anos. Além do pole, o local também tem aulas de dança do ventre. Ao todo, até o momento deste estudo, o estabelecimento apresentava 64 alunas matriculadas, 45 eram praticantes de pole dance.

Para a realização desta pesquisa houve a participação de 30 mulheres, que foram escolhidas de forma aleatória. Os critérios de inclusão das participantes foram mulheres matriculadas e que frequentassem as aulas de pole dance regularmente, por no mínimo três meses, no studio/academia selecionado para a aplicação do questionário. Os critérios de exclusão foram mulheres que não fizessem aula de pole dance regularmente ou que não desejassem participar do estudo. A pesquisa foi aprovada pelo Comitê de Ética em Pesquisa Envolvendo Seres Humanos, sob parecer $\mathrm{n}^{\circ}$. 1.093.322/2015-COMEP/ UNICENTRO.

A coleta foi realizada na academia onde as mulheres praticavam o pole dance, antes ou após as aulas. Para a coleta de dados foi utilizado, inicialmente, um questionário socioeconômico, elaborado pelos pesquisadores, que apresentava questões sobre escolaridade, profissão, estado civil, idade, tempo 
de prática de pole dance e prática de outros tipos de atividades físicas. Posteriormente, foi aplicado o questionário Exercise Motivations Inventory-2 (EMI-2). Originalmente idealizado na língua inglesa (MARKLAND; HARDY, 1993; MARKLAND; INGLEDEW, 1997), traduzido e validado para o idioma português por Alves e Lourenço, ${ }^{12}$ o EMI2 recebeu a denominação de Questionário de Motivação para o Exercício (QME). De acordo com seus idealizadores, o EMI-2 resulta em um instrumento mais válido que os demais, considerando que contempla uma maior variedade de motivos. É composto por 51 itens, disponibilizado em uma escala Lickert de 6 pontos $(0=$ "nada verdadeiro" até 5 = "muito verdadeiro"), agrupados em 14 fatores motivacionais e 5 dimensões: (a) psicológicas; (b) interpessoais; (c) saúde; (d) estética; (e) condições físicas.

Para a análise dos dados da pesquisa, foi utilizada a estatística descritiva. A análise das respostas foi realizada mediante o cálculo da média e desvio padrão dos itens correspondentes a cada fator motivacional. Dessa forma, os fatores com valores médios superiores a " 2,5 " foram considerados os principais fatores de motivação para a prática de pole dance.

\section{RESULTADOS E Discussão}

A amostra foi constituída por $30 \mathrm{mu}-$ lheres com idade média de 31,5 anos e com média de prática regular de pole dance de 22,6 meses. A maioria relatou, por meio do questionário socioeconômico, serem casadas $(56,6 \%)$, apresentarem carga horária de trabalho maior que seis horas diárias (73,3\%) e possuírem ensino superior (66\%).
A respeito de fazer outro tipo de atividade física, 50\% afirmaram que realizam. Entre as mais citadas foram caminhadas, dança e pilates. Também foram questionadas sobre a desistência da prática de exercícios físicos anteriores e os motivos mais citados foram a falta de tempo e atividade muito repetitiva.

O Gráfico 1 apresenta os resultados obtidos no questionário EMI-2, apontando os motivos que levam as mulheres à prática do pole dance.

Dos dez fatores motivacionais considerados no estudo, sete deles, sendo condição física, diversão e bem-estar, controle de estresse, prevenção de doenças, aparência física, controle do peso corporal e afiliação, apresentaram valores médios superiores ao ponto de corte de 2,5; portanto, podem ser considerados relevantes. No entanto, os outros três fatores, sendo competição, reabilitação e reconhecimento social, exibiram médias menores que o ponto de corte de 2,5 e, desse modo, podem ser vistos como fatores não relevantes de motivação para a prática de pole dance.

O estudo realizado por Balbinotti e Capozzoli ${ }^{13}$ avaliou a motivação à prática regular de atividade física com praticantes em academias de ginástica, as mulheres motivam-se por controle de estresse, saúde, e estética, enquanto os menores valores foram encontrados na variável competitividade. Tais resultados corroboram com os achados do presente estudo.

Cardoso et al. ${ }^{14}$ investigaram a motivação em mulheres praticantes de exercícios físicos em academias. Mais evidentes foram também os fatores motivacionais de condicionamento físico, estética e saúde, e menos evidentes os motivos interpessoais. 
Gráfico 1 - Fatores motivacionais para o pole dance.

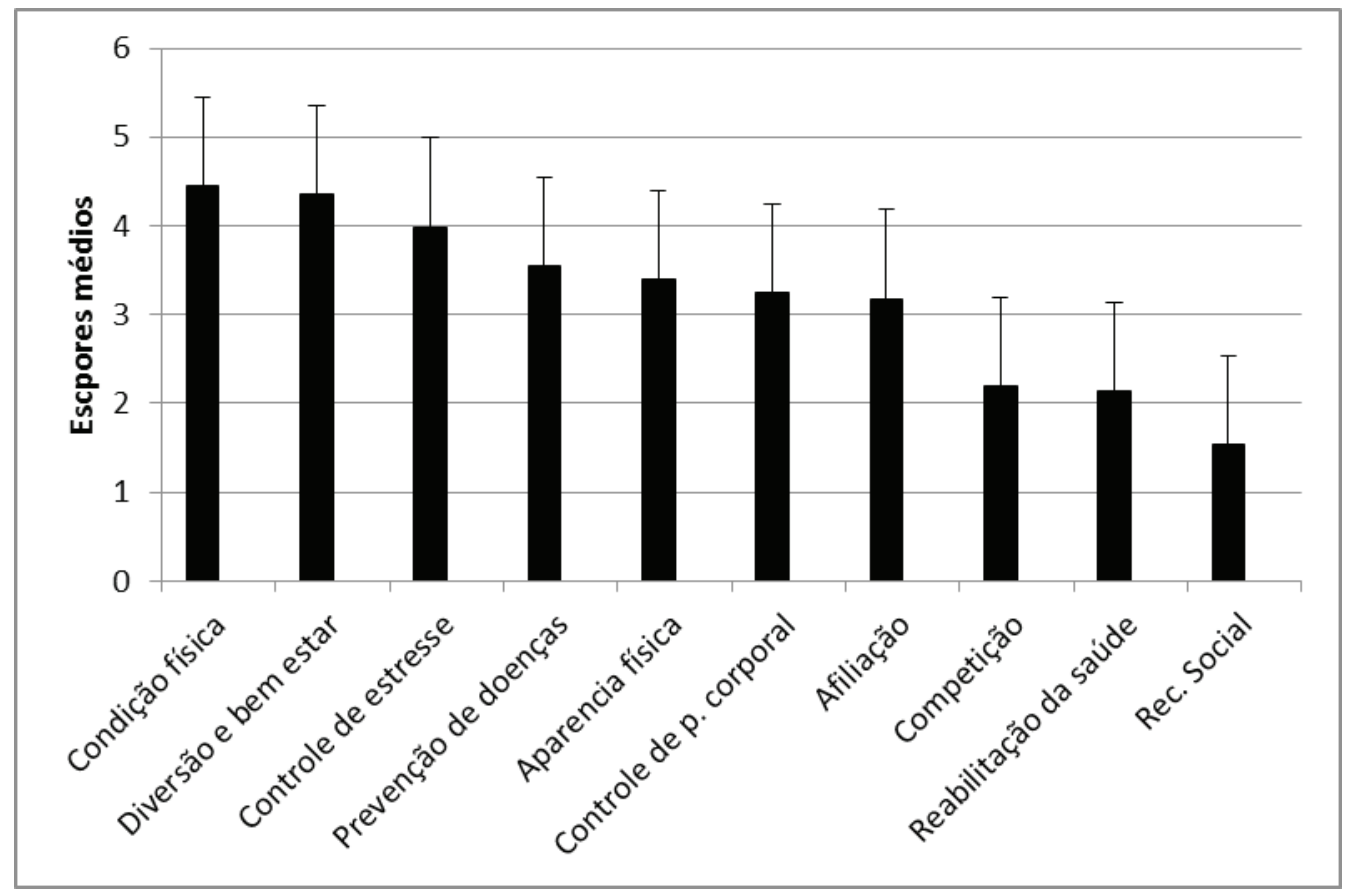

Deschamps et al. ${ }^{15}$ avaliaram os motivos para a prática físico-desportiva de universitários de educação física e mostraram que as mulheres se preocupam mais com a questão da imagem, saúde e busca de aventura. Quando o assunto é motivação, elas empregam o mesmo grau de importância entre os fatores, o que pode estar relacionado aos padrões e ideais de beleza feminina impostos pela sociedade.

Oliveira ${ }^{16}$ analisou a motivação de praticantes de escalada esportiva indoor e verificou que melhorar o condicionamento físico foi considerado um motivo muito importante pelos participantes do estudo. Em relação aos motivos de menor relevância para os escaladores entrevistados, destaca-se o aumento de reconhecimento social. Grande parte dos participantes da pesquisa não se motiva a praticar escalada esportiva indoor pelo prestígio ou situação hierárquica que ele pode representar nesse grupo social.

Estudo realizado por Deschamps e Domingues ${ }^{17}$ apresentou os motivos e benefícios psicológicos que levam os indivíduos dos sexos masculino e feminino a praticarem o ciclismo indoor. Os principais resultados obtidos para a prática do exercício foram o prazer pela atividade, a melhora da estética, melhora no condicionamento físico e qualidade de vida. Os indivíduos também relataram que o ciclismo indoor auxilia no combate ao estresse e durante a prática ocorre um relaxamento mental.

Truccolo et al. ${ }^{18}$ investigaram os fatores motivacionais de adesão a grupos de corrida. As razões mais importantes para as mulheres aderirem a um programa de corrida supervisionado foram a melhora no con- 
dicionamento físico, a saúde, o aumento da autoestima e a aparência física.

Apesar das diferenças entre as modalidades apresentadas e o pole dance, também encontramos pontos semelhantes sobre a motivação para a prática. As modalidades possuem condicionamento físico como principal fator motivacional, relacionado também com a saúde, qualidade de vida e bem-estar. $O$ fator estético não parece relevante para os praticantes de escalada indoor que participaram da pesquisa, já que o mesmo não foi citado. De modo diferente do pole, do ciclismo indoor e grupos de corrida, em que o fator estético, segundo os resultados apresentados, possui relevância para a adesão das modalidades. Comparando os fatores de menor importância, assim como no pole, a escalada indoor também tem o reconhecimento social como não relevante para a prática.

Caruzzo et al. ${ }^{19}$ analisaram os fatores motivacionais que levam adultos e idosos à prática de hidroginástica em uma cidade da região norte do Paraná, utilizando o questionário EMI-2. Os resultados demonstraram maior motivação na prevenção de doenças, melhora da condição física, da aparência física e do controle do estresse. É relevante destacar que muitos dos idosos foram motivados a engajar-se na hidroginástica por orientação médica.

A análise da motivação para a prática de hidroginástica, apresentada no estudo citado, utilizou o mesmo questionário aplicado para esta pesquisa, indicando os fatores prevenção de doenças, condicionamento físico e controle de estresse entre os principais motivos que levam à prática. Tais fatores também aparecem entre os motivos com média mais alta e considerados relevantes para a prática de pole dance.

Costa et al. ${ }^{20}$ investigaram a motivação de atletas de ginástica rítmica e corredoras de rua, identificando o fator motivacional predominante. Foi constatada a "motivação interna para atingir um objetivo" na modalidade de ginástica rítmica e a "motivação interna relacionada com experiências estimulantes" para corredoras de rua. O estudo demonstrou que os participantes da pesquisa são motivados intrinsecamente, tanto para corrida de rua quanto para ginástica rítmica. Para a prática de pole dance foi constatado tanto motivos internos quantos externos, pois condição física e controle de estresse são tidos como variáveis extrínsecas, enquanto diversão e bem-estar são apresentados como fatores intrínsecos.

Conltri e Piovani ${ }^{21}$ realizaram um estudo para identificar os aspectos motivacionais que levam as pessoas a praticarem aulas de dança. Evidenciou-se que as dimensões apresentadas foram prazer, saúde, sociabilidade, estética e controle de estresse. Ressalta-se que o prazer e a busca pela saúde na prática de dança merecem destaque, pois a dimensão prazer, ao responder a fatores intrínsecos de motivação dos sujeitos, tende a promover a aderência à prática de exercício físico. A busca pela saúde, tanto do ponto de vista interno como externo, demonstra que a dança não se limita a uma modalidade técnica, mas oferece também uma possibilidade para promoção de saúde.

$\mathrm{O}$ estudo mencionado, sobre a motivação para a adesão às aulas de dança, deu principal ênfase nas dimensões prazer e saúde, da mesma forma que a presente pesquisa a respeito do pole dance. Os fatores condi- 
cionamento físico e controle de estresse podem estar atrelados à dimensão saúde, enquanto a diversão e o bem-estar podem ser relacionados com a dimensão prazer.

Fernandes ${ }^{22}$ realizou um estudo a respeito do motivo de aderência de mulheres à prática regular de aulas de pole dance na cidade de Criciúma-SC. A metodologia abordada foi de entrevistas abertas com as praticantes. Os resultados mostram que as mulheres procuraram a prática de pole dance por curiosidade sobre as aulas, citando que os motivos que as levam a continuarem a prática são variados, como estética e autoconfiança. Como a metodologia abordada difere da utilizada no presente estudo, não é possível realizar uma comparação clara. Mesmo assim, o trabalho realizado pelos pesquisadores em Criciúma faz-se relevante, considerando que não foram encontradas outras pesquisas acerca da motivação para prática de pole dance. A literatura sobre o pole ainda é escassa e foram encontrados apenas estudos referentes à revisão histórica, preconceito e gênero relacionados à prática da modalidade.

\section{CONSIDERAÇõES FINAIS}

A presente pesquisa teve por objetivo analisar os motivos que levam mulheres à prática do pole dance como atividade física. De modo geral, os resultados apontaram que os principais motivos foram "condicionamento físico", "diversão e bem-estar" e "controle de estresse", enquanto os fatores "competição", "reabilitação física" e "reconhecimento social" assinalaram menor relevância. Evidenciou-se, dessa forma, que o que leva as mulheres participantes da pesquisa à prática do pole dance tem maior relação com a melhora/manutenção da saúde e com o prazer que o exercício da modalidade pode oferecer. O caráter competitivo ou reabilitador do pole, além do prestígio social que a ele possa estar atrelado, não são fatores de grande importância para as pesquisadas.

Apesar de recente e publicações sobre o tema serem escassas, o pole dance tem se desenvolvido no campo fitness com sua inserção em academias e estúdios especializados, bem como, pelo aumento do número dos cursos de habilitação profissional na área da modalidade. Tem se desenvolvido também como esporte, na realização crescente de campeonatos nacionais e mundiais.

Sendo assim, a presente pesquisa possibilitou ampliar os estudos sobre a prática do pole dance, averiguando os fatores motivacionais vinculados a sua prática por mulheres. Para novos estudos, sugere-se a realização de análises relacionadas a outros fatores psicológicos que podem influenciar na prática do pole, como a autoimagem, estado de humor, traços de perfeccionismo etc. Além destes, os efeitos que a prática regular de pole pode oferecer na melhora dos aspectos físico-motores e sociais de seus praticantes também podem ser considerados em estudos futuros. 


\section{REFERÊNCIAS}

1. Santos, AC. Benefícios do pole dance sobre a aptidão física: Uma revisão teórica. In: Encontro de Iniciação Científica da Fifapa, Paranavaí, 3, 2012, Anais... Paranavaí, 2012, p. 1-4.

2. Santos, SC; Knijnik, JD. Motivos de adesão à prática de atividade física na vida adulta intermediária. Rev Mackenzie de Ed Fís e Esp. 5 (1): 23-34, 2006.

3. Weinberg, RS.; Gould, D. Fundamentos da Psicologia do Esporte e do Exercício, 2. ed. São Paulo: Artmed, 2001.

4. Samulski, D. Psicologia do esporte: teoria e aplicação prática. Belo Horizonte: Imprensa UFMG, 1995.

5. Balbinotti, et al. Motivação à prática regular de atividades físicas e esportivas: um estudo comparativo entre estudantes com sobrepeso, obesos e eutróficos. Motriz, Rio Claro, v. 17, n. 3, p. 384-394, jul./set. 2011.

6. Samulski, D. Psicologia do esporte: conceitos e novas perspectivas, 2. ed. São Paulo: Manole, 2009.

7. Samulski, D. Psicologia do Esporte, 1. São Paulo: Manole, 2002.

8. Borges, NS. Possibilidade da prática de ensino no pole dance: análise do movimento bandeira. Trabalho de conclusão de curso (graduação em Educação Física). Universidade Paulista (UNIP). São Paulo, 2015.

9. Parizzi, L. Pole Dance Brasil - História do Pole Dance. Disponível em: <http://www.poledancebrasil.com.br/pole-dance-a-historia/ > . Acesso em: 20 abr. 2017.

10. Cirque du Soleil, 2013. Disponível em: https://m.cirquedusoleil.com/pt/about/,intro.aspx acesso em: 25 jan. 2017.

11. Silva, EL; Menezes, EM. Metodologia da pesquisa e elaboração de dissertação, 4. ed. Florianopolis-SC: UFSC, 2005.

12. Alves, J., Lourenço, A. Tradução e adaptação do questionário de motivação para o exercício. Desporto, Investigação e Ciência, 2, 3-23, 2003.

13. Balbinotti, MAA.; Capozzoli, CJ. Motivação à prática regular de atividade física: um estudo exploratório com praticantes em academias de ginástica. Rev. bras. Educ. Fís. Esp. São Paulo, v. 22, n. 1, p. 63-80, jan./mar. 2008.

14. Cardoso J.; Chrun R. D.; et al. Motivação em mulheres praticantes de exercícios físicos em academias de Mandaguari-Pr. In: congresso internacional de pedagogia do esporte, Maringá, 6., 2015. Anais... Maringá: 2015, p. 4-6.

15. Deschamps, SR.; Martins, DS.; Paroli, R.; Domingues Filho, LA. Motivos, interesses e atitudes para a prática físico-desportiva dos universitários de educação física. Rev Bras de Ed Fís e Esp, v. 23, n. 1, p. 51-59, 2009.

16. Oliveira, BVM. Análise da motivação para praticantes de escalada esportiva indoor no município de Belo Horizonte-MG. 2010. Trabalho de conclusão de curso (Graduação em Educação Física), Universidade Estadual de Minas Gerais. Belo Horizonte. 2010.

17. Deschamps, SR; Domingues Filho, LA. Motivos e benefícios psicológicos que levam os indivíduos dos sexos masculino e feminino a praticarem o ciclismo indoor. R. bras. Ci e Mov. 2005; 13 (2): 27-32.

18. Truccolo, AB.; Maduro, PA.; et al. Fatores motivacionais de adesão a grupos de corrida. Motriz, Rio Claro, v. 14, n. 2, p. 108-114, abr./jun. 2008.

19. Caruzzo, MN.; Cardoso, PT.; et al. Motivos que levam a pratica da hidroginástica. In: Congresso internacional de pedagogia do esporte, Maringá, 6., 2015. Anais... Maringá, 2015. 
20. Costa, RC; Silva, AK; et al. Motivação entre atletas de ginástica rítmica e corredoras de rua: um estudo comparativo. In: Congresso internacional de pedagogia do esporte, Maringá, 6., 2015. Anais... Maringá, 2015, p. 1.

21. Coltri, ML.; Piovani, SGV. Motivação para a prática de dança. In: Congresso internacional de pedagogia do esporte, Maringá, 6., 2015. Anais... Maringá, 2015.

22. Fernandes, MJ. Motivo na aderência de mulheres a pratica regular de aulas de pole dance na cidade de Criciúma-SC. Trabalho de conclusão de curso (Graduação em Educação Física). Universidade do Extremo Sul de Santa Catarina. Criciúma, 2012.

\section{DADOS DOS AUTORES}

\section{Elis Regina Lopes}

Graduada em Educação Física Bacharelado pela Universidade Estadual do Centro-Oeste (UNICENTRO). Empresária e instrutora de Pole Dance. Guarapuava/PR - Brasil. elislopes92@hotmail.com

\section{Thiago Emanuel Medeiros}

Mestre em Ciências do Movimento Humano pela Universidade do Estado de Santa Catarina (UDESC). Doutorando do Programa de Pós-Graduação em Ciências do Movimento Humano na mesma instituição. Florianópolis/SC - Brasil. temedeiros@yahoo.com.br

\section{Veronica Volski Mattes}

Mestre em Educação pela Universidade Estadual do Centro-Oeste (UNICENTRO). Docente do Departamento de Educação Física na mesma instituição. Guarapuava/PR - Brasil. vero_edf@hotmail.com

Submetido em: 14-3-2018

Aceito em: 13-5-2019 